\title{
Chronic Partial Rupture of Distal Biceps Tendon in an Adolescent-A Case Report
}

\author{
Ashwin Hampole, M. Mukarram Sheikh, Anugayathri Jawahar, Aruna Vade \\ Loyola University Medical Center, Maywood, USA \\ Email: anugayathri@gmail.com
}

Received 2 April 2014; revised 1 May 2014; accepted 20 May 2014

Copyright (C) 2014 by authors and Scientific Research Publishing Inc.

This work is licensed under the Creative Commons Attribution International License (CC BY). http://creativecommons.org/licenses/by/4.0/

c) (i) Open Access

\begin{abstract}
Distal biceps tendon rupture accounts for only 3\% - 10\% of all biceps tendon injuries. The majority of distal biceps tendon injuries are from complete rupture. It is especially rare to diagnose partial rupture of the distal biceps tendon. Our case profiles a chronic partial tear of the distal biceps tendon in a pediatric patient.
\end{abstract}

\section{Keywords}

Biceps Tendon, Partial Tear, Distal Biceps Tendon, Pediatric, Chronic Partial Tear

\section{Introduction}

Injury with tear of the distal biceps tendon is rare, accounting for only $3 \%-10 \%$ of all biceps tendon injuries, and has an incidence of 1.2 per 100,000 patients per year [1]-[10]. Tears of the distal biceps brachii tendon are less common than tears of the proximal biceps brachii tendon [7] [11]. They occur most frequently in the older adult population aged 40 - 60 years and are more common in men [5] [6] [8]-[12]. Complete tears are relatively common in men and usually traumatic in origin, whereas partial tears of distal biceps tendon are more common in women and usually have an insidious onset [4] [10] [11]. The result for gender disparity in the frequency of distal biceps tendon tear remains unclear [10]. There are no documented cases of distal biceps tendon injuries in pediatric population.

The diagnosis of biceps injury is most commonly made clinically when physical examination following an elbow injury shows swelling and tenderness in the antecubital fossa, supination weakness with elbow flexion, grip strength deficit and biceps tendon defect distally [1]. Imaging is not generally required for diagnosis but may help to clarify atypical presentations and differentiate between partial ruptures and other antecubital fossa pathologies [1] [2] [13] [14]. 


\section{Case Report}

A 13-year-old left hand dominant male initially presented for evaluation of 2 days history of anterior elbow pain and limited movements of the left elbow. Physical examination of the left elbow was normal. Patient did not recall any history of trauma. The left elbow radiographs revealed probable elevation of the fat plane anterior to the proximal radius, raising suspicion for an occult fracture of the proximal radius. Short-term follow up radiographs of the elbow were recommended. However, since the physical examination was normal, the physician diagnosed it to be muscular pain, treated him with NSAIDs with instructions to consult an orthopedic surgeon if symptoms worsened. 10 months later the patient presented to the hospital with history of progressive left anterior elbow pain and gradual decrease in elbow movements for last 2 months. The pain was worse with activity and improved with rest. Examination revealed deformity of the left proximal forearm with a palpable mass at the bicipital tuberosity associated with limitation of supination. The patient was not able to pronate or reach neutral position of the elbow. Full elbow extension was possible. Radial pulse was normal distally. There was full range of movements in the left hand. Elbow radiographs at this time demonstrated a deformity of the proximal radius with circumferential consolidated periosteal reaction in the proximal radius centered at the radial tuberosity consistent with a chronic healing fracture of the radial tuberosity (Figure 1). Because of a palpable mass in the anterior proximal forearm, a contrast-enhanced MRI and CT of the elbow was performed to evaluate for soft tissue and bone abnormalities.

The MRI showed thickening of the distal biceps tendon adjacent to its insertion on the radial tuberosity with intermediate signal on T1W and hyperintense signal on T2W images (Figure 2(a)). There was fluid collection in the bicipital bursa (Figure 2(b)) with thickened enhancing synovium (Figure 2(c)). Periosteal reaction and bone marrow edema in the proximal radius (Figure 2(b)) was associated with cortical erosion of the radial tuberosity adjacent to the insertion of the biceps tendon. The referring physician requested a CT of the elbow to further evaluate the radius abnormality. CT confirmed the cortical erosion seen on MRI at the anteromedial aspect of the radial tuberosity at the site of the biceps tendon insertion with preservation of intramedullary trabecular architecture (Figure 3(a), Figure 3(b)). CT also confirmed the cortical thickening in the region of radial tuberosity extending into the proximal shaft of radius (Figure 3(b)). There was no evidence of endosteal scalloping to suggest a lytic bony lesion. A diagnosis of biceps partial tear with stress related changes in the radius and bicipital bursa was made.

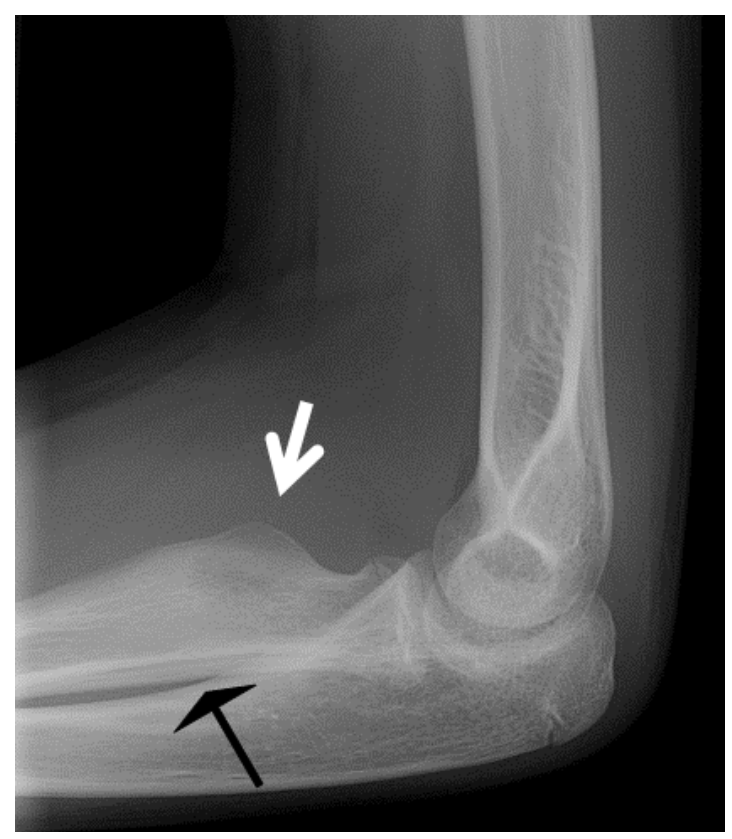

Figure 1. Lateral radiograph of left elbow 10 months after initial presentation shows a new deformity at the proximal radius with thickening of anterior cortex (white arrow) and consolidated periosteal reaction posteriorly (black arrow), consistent with a chronic healing fracture. 


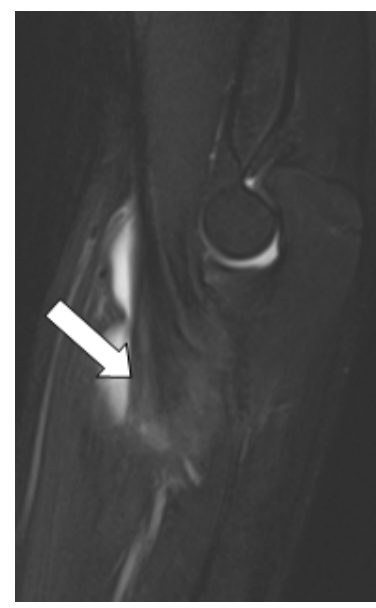

(a)

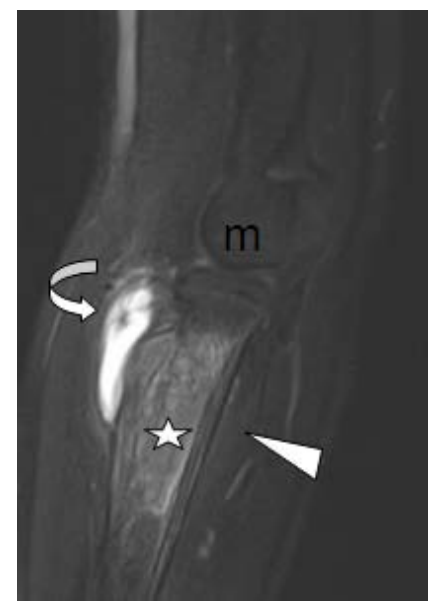

(b)

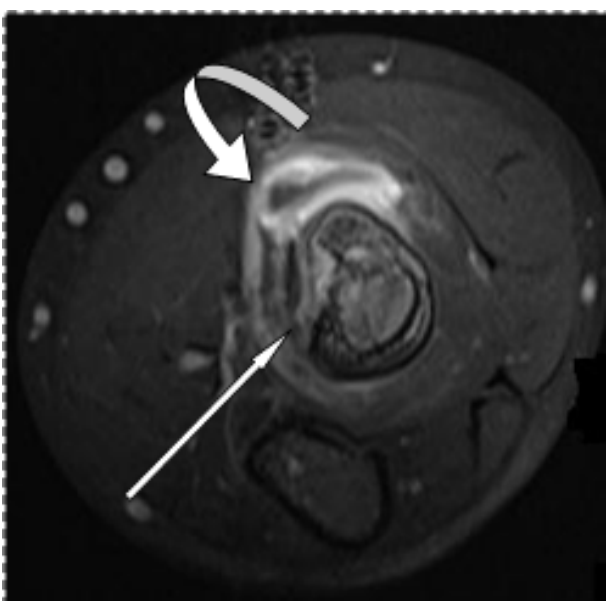

(c)

Figure 2. Sagittal T2W (a) and (b), and axial T1 post-contrast (c) MR images of the elbow show abnormal hyperintense signal of the distal biceps tendon near its insertion on the radial tuberosity (white arrows (a)) representing a partial biceps tendon rupture, fluid in the bicipital bursa (curved arrow (b)) with synovial enhancement (curved arrow (c)), bone marrow edema in proximal radius (b), periosteal reaction in the posterior radius (arrow head (b)) and cortical erosion in the radial tuberosity adjacent to the biceps tendon insertion (thin arrow (c)).

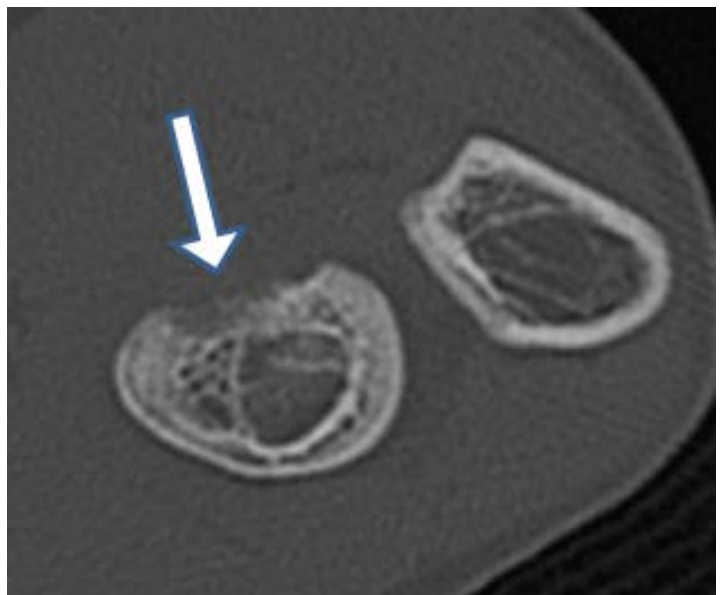

(a)

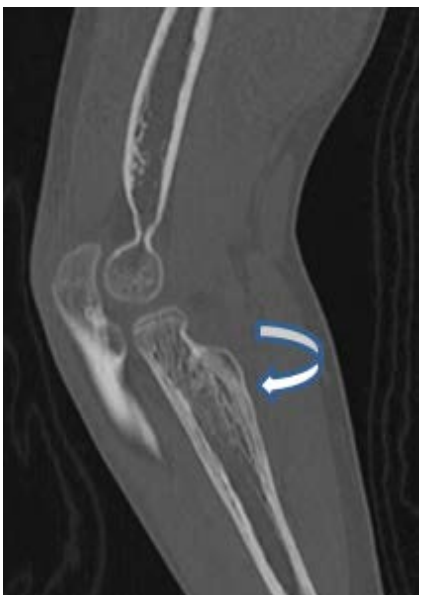

(b)

Figure 3. CT axial and sagittal reformatted images of the elbow demonstrate cortical erosion at the anteromedial aspect of the radial tuberosity near the biceps insertion (straight arrow (a)) and cortical thickening and preservation of the intramedullary trabecular architecture (curved arrow (b)).

The patient was managed non-operatively with anti-inflammatory medication. His elbow pain subsided and he regained elbow motion with physical therapy after 4 months and the physician deferred follow up radiograph.

\section{Discussion}

The biceps brachii muscle originates at the scapula with its long head from the supraglenoid tubercle and with its short head from the corocoid process of scapula. The muscle bellies of the long and short heads unite as they descend on the anterior aspect of upper arm and separate proximal to the level of elbow joint to form incompletely united muscle bellies with their respective tendons. The deep tendinous fibers of both heads form the main tendon of distal biceps, which enters the lateral aspect of the cubital fossa to attach at the radial tuberosity [7]. The two independent muscle bellies with a bifurcated distal biceps tendon probably allows functional independence and isolated rupture of each portion [15]. The biceps tendon is normally separated from the anterior portion of the radial tuberosity by a bursa [12]. There are three vascular zones within the distal biceps brachii 
tendon. The proximal zone 1 is supplied by the brachial artery, zone 2 is approximately $2 \mathrm{~cm}$ proximal to insertion and represents a relatively hypovascular zone and distal zone 3 is supplied by the posterior recurrent artery [16]. Seiler et al proposed that the mechanical impingement of the muscle during rotation and the relatively hypovascular zone 2 may contribute to distal biceps tendon tears [11] [16].

The mechanism of injury to the distal biceps brachii tendon in majority of the patients is chronic degenerative changes in the tendon that disrupt the normal architecture of the tendon and makes it more vulnerable to rupture when sudden flexion of elbow or sudden eccentric contraction of the biceps brachii muscle is applied against resistance [1] [4]-[6] [11]. In light of these predisposing factors for a distal biceps tendon injury, it is exceedingly rare to see distal biceps tendon injury in the young population. Partial tears of distal biceps tendon may be traumatic or attritional and usually involve only one of the components of biceps tendon (short head or long head) [4]-[6]. Our patient did not recall any history of trauma.

Partial tears of the distal biceps tendon are not dramatic in presentation and generally present with anterior elbow pain which was the presentation in our patient [17]. Partial tears involving the long head of biceps tendon usually presents with a typical Popeye sign which is a soft tissue lump over the anterior aspect of the middle third of arm, suggesting retraction of belly of biceps which was not seen in our patient [4]. Sometimes the symptoms of partial biceps tendon tear may even mimic or co-exist with conditions like bicipital tendinosis, leading to missed or delay in the diagnosis of chronic distal biceps tendon tear [17].

The study by Festa et al. showed MRI to have a sensitivity of $59.1 \%$, specificity and positive predictive value of $100 \%$ in diagnosing partial tears of distal biceps tendon [17]. Another study by Fitzgerald et al showed that axial MRimages were more valuable in pre-operative grading of distal biceps tendon injury and that there was $100 \%$ agreement between MRI and surgical findings [18]. MRI appearance of partial tears include intratendinous high signal intensity on T2-weighted images, bicipito-radial bursitis, fluid within the biceps tendon sheath, thinning or thickening of the tendon and increased bone marrow edema at the tendon insertion site [5] [14] [17]. Presence of increased intratendinous signal intensity on T2-weighted images can also be seen in biceps tendinopathy but without the bone marrow changes and bicipital bursitis. In addition, partial distal biceps tendon tears and tendinopathy may both present with increased T2 signal intensity without intratendinous fluid as seen in our patient [17]. Initially the referring physician managed the patient for muscular pain. If an MRI had been obtained at the time of initial presentation, presence of bone marrow edema in the proximal radius could have helped differentiate a partial tear of the distal biceps tendon frombiceps tendinopathy. Rarely, partial rupture of distal biceps tendon can present as a pseudo-tumor due to a slowly progressive, localized proliferative synovitic process following injury [5]. Our patient presented with a palpable mass in the region of the radial tuberosity which could have been due to radial tuberosity hypertrophy and associated bicipital bursitis with synovitis. Given the rarity of distal biceps tendon rupture, particularly in the pediatric population, there was clinical concern in our patient for malignancy of the proximal radius which was effectively ruled out by CT and MRI.

Plain radiographs if obtained in patients with distal biceps tendon injury rarely show an avulsion of the radial tuberosity [9]. It is likely that our patient may have had a partial tear of the biceps tendon at the time of initial presentation that caused chronic stress on the radial tuberosity leading to hypertrophy of the radial tuberosity with bone marrow edema and bicipital synovitis. The other possibility is ongoing stress to the proximal radius from a healing missed radial tuberosity avulsion fracture leading to hypertrophy of the radial tuberosity, bone marrow edema and periosteal reaction. Hypertrophy of the radial tuberosity is known to cause impingement of the distal biceps tendon, particularly in a full forearm pronation, leading to tendinopathy or low grade partial distal biceps tears [1] [17].

Partial tears of the distal biceps brachii tendon and biceps tendinopathy are usually managed with conservative measures like rest and physiotherapy. Patients who do not improve with non-operative measures are treated with surgery which may include either debridement or completion of the tear with tendon reinsertion [5] [13].

\section{Conclusion}

In summary, injury of the distal biceps brachii tendon should be considered in a pediatric patient complaining of anterior elbow pain with limited elbow mobility and normal elbow radiographs. MRI can characterize biceps tendinosis and partial tears and help in timely and appropriate clinical management avoiding subsequent radial deformity and bicipital synovitis. 


\section{References}

[1] Vidal, A.F., Drakos, M.C. and Allen, A.A. (2004) Biceps Tendon and Triceps Tendon Injuries. Clinics in Sports Medicine, 23, 707-722.

[2] Miyamoto, R.G., Elser, F. and Millett, P.J. (2010) Distal Biceps Tendon Injuries. The Journal of Bone and Joint Surgery, 92, 2128-2138. http://dx.doi.org/10.2106/JBJS.I.01213

[3] Dirim, B., Brouha, S.S., Pretterklieber, M.L., et al. (2008) Terminal Bifurcation of the Biceps Brachii Muscle and Tendon: Anatomic Considerations and Clinical Implications. American Journal of Roentgenology, 191, W248-W255. http://dx.doi.org/10.2214/AJR.08.1048

[4] Tagliafico, A., Michaud, J., Capaccio, E., Derchi, L.E. and Martinoli, C. (2010) Ultrasound Demonstration of Distal Biceps Tendon Bifurcation: Normal and Abnormal Findings. European Radiology, 20, 202-208. http://dx.doi.org/10.1007/s00330-009-1524-1

[5] Bradshaw, A.R., Sandow, M.J. and Clayer, M.T. (2011) Distal Biceps Tendon Partial Tear Presenting as a Pseudotumor. Journal of Shoulder and Elbow Surgery, 20, e14-e17. http://dx.doi.org/10.1016/j.jse.2010.08.011

[6] Cain, R.A., Nydick, J.A., Stein, M.I., Williams, B.D., Polikandriotis, J.A., et al. (2012) Complications Following Distal Biceps Repair. JHS, 37A, 2112-2117.

[7] Dirim, B., Brouha, S.S., Pretterklieber, M.L., Wolff, K.S., Frank, A., et al. (2008) Terminal Bifurcation of the Biceps Brachii Muscle and Tendon: Anatomic Considerations and Clinical Implications. AJR, 191, W248-W255.

[8] Webley, J. (2012) Ruptures Distal Biceps Tendon. The Journal of Emergency Medicine, 43, e471-e472. http://dx.doi.org/10.1016/j.jemermed.2011.06.052

[9] Sherman, S.C. and Afifi, N. (2012) Distal Biceps Tendon Rupture. The Journal of Emergency Medicine, 43, e469-e470. http://dx.doi.org/10.1016/j.jemermed.2011.06.041

[10] Jockel, C.R., Mulieri, P.J., Belsky, M.R. and Leslie, B.M. (2010) Distal Biceps Tendon Tears in Women. Journal of Shoulder and Elbow Surgery, 19, 645-650. http://dx.doi.org/10.1016/j.jse.2010.01.015

[11] Da Gama Lobo, L., Fessell, D.P., Miller, B.S., Kelly, A., Lee, J.Y., et al. (2013) The Role of Sonography in Differentiating Full versus Partial Distal Biceps Tendon Tears: Correlation with Surgical Findings. AJR, 200, 158-162. http://dx.doi.org/10.2214/AJR.11.7302

[12] Strauch, R.J. (1999) Biceps and Triceps Injuries of the Elbow. The Orthopedic clinics of North America, 30, 95-107. http://dx.doi.org/10.1016/S0030-5898(05)70064-X

[13] Kijowski, R., Tuite, M. and Sanford, M. (2005) Magnetic Resonance Imaging of the Elbow. Part II: Abnormalities of the Ligaments, Tendons, and Nerve. Skeletal Radiology, 34, 1-18. http://dx.doi.org/10.1007/s00256-004-0854-y

[14] Falchook, F.S., Zlatkin, M.B., Erbacher, G.E., Moulton, J.S., Bisset, G.S., et al. (1994) Rupture of the Distal Biceps Tendon: Evaluation with MR Imaging. Radiology, 190, 659-663.

[15] Cho, C.-H., Song, K.-S., Choi, I.-J., Kim, D.-K., Lee, J.-H., et al. (2011) Insertional Anatomy and Clinical Relevance of the Distal Biceps Tendon. Knee Surgery, Sports Traumatology, Arthroscopy, 19, 1930-1935. http://dx.doi.org/10.1007/s00167-011-1586-x

[16] Seiler 3rd, J.G., Parker, L.M., Chamberland, P.D., Sherbourne, G.M. and Carpenter, W.A. (1995) The Distal Biceps Tendon: Two Potential Mechanisms Involved in Its Rupture-Arterial Supply and Mechanical Impingement. Journal of Shoulder and Elbow Surgery, 4, 149-156. http://dx.doi.org/10.1016/S1058-2746(05)80044-8

[17] Festa, A., Mulieri, P.J., Newman, J.S., Spitz, D.J. and Leslie, B.M. (2010) Effectiveness of Magnetic Resonance Imaging in Detecting Partial and Complete Distal Biceps Tendon Rupture. JHS, 35A, 77-83.

[18] Fitzgerald, S.W., Curry, D.R., Erickson, S.J., Quinn, S.F. and Friedman, H. (1994) Distal Biceps Tendon Injury: MR Imaging Diagnosis. Radiology, 191, 203-206. 